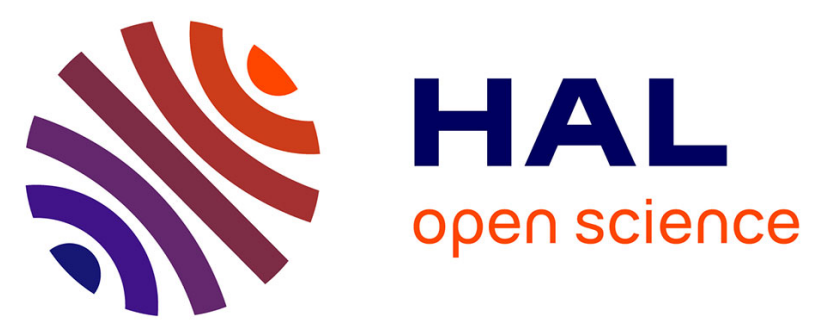

\title{
Diversity in Shallow Water Environments Using Blind Time-Frequency Separation Techniques
}

Bertrand Gottin, Jun Zhang, Antonia Papandreou-Suppappola, Cornel Ioana

\section{To cite this version:}

Bertrand Gottin, Jun Zhang, Antonia Papandreou-Suppappola, Cornel Ioana. Diversity in Shallow Water Environments Using Blind Time-Frequency Separation Techniques. ACSSC 2007 - 41st Annual Asilomar Conference on Signals Systems and Computers, Nov 2007, Pacific Grove, United States. hal-00326362

\section{HAL Id: hal-00326362 https://hal.science/hal-00326362}

Submitted on 2 Oct 2008

HAL is a multi-disciplinary open access archive for the deposit and dissemination of scientific research documents, whether they are published or not. The documents may come from teaching and research institutions in France or abroad, or from public or private research centers.
L'archive ouverte pluridisciplinaire HAL, est destinée au dépôt et à la diffusion de documents scientifiques de niveau recherche, publiés ou non, émanant des établissements d'enseignement et de recherche français ou étrangers, des laboratoires publics ou privés. 


\title{
Diversity in Shallow Water Environments Using Blind Time-Frequency Separation Techniques
}

\author{
Bertrand Gottin $^{\dagger}$, Jun Zhang ${ }^{\ddagger}$, Antonia Papandreou-Suppappola ${ }^{\ddagger}$ and Cornel Ioana ${ }^{\dagger}$ \\ ${ }^{\dagger}$ GIPSA-LAB, INPG/ENSIEG, Grenoble, France \\ ${ }^{\ddagger}$ SenSIP Center, Department of Electrical Engineering, Arizona State University, Tempe, AZ, USA \\ E-mail: Bertrand.Gottin@asu.edu, Jun.Zhang.EE@asu.edu, papandreou@asu.edu,cornel.ioana@gipsa-lab.inpg.fr
}

\begin{abstract}
The shallow water environment can be characterized as a time-dispersive system whose time-varying impulse response can be expressed as a superposition of time-frequency components with dispersive structures. In this paper, a blind timefrequency processing technique is employed to separate these components without knowledge of environmental parameters. This technique is based on first approximating the time-frequency structures of the received signal, and then designing separation filters based on time-frequency warping techniques. Based on this method, a receiver is developed to exploit the diversity of the channel and to improve communications performance.
\end{abstract}

\section{INTRODUCTION}

The shallow water acoustic environment is a linear timevarying (TV) dispersive system that can shift different frequencies by different amounts in time [1] due to the transmitted waveform's interactions with the ocean bottom and surface. This dispersive effect can severely limit the performance of underwater acoustic applications such as sonar and communications. It can be shown that the propagation characteristics of such an environment can be determined by specific nonlinear functions that define the dispersion in this environment and provide a means of modeling the environment according to how it distorts the transmitted signal. Thus, signals can be used to exploit the potential diversity suggested by the model when the receiver is appropriately designed to match these nonlinear functions.

In [1], a characterization of shallow water was considered that matched dispersive signal transformations on the transmitted waveforms and was successfully used for shallow water communications to obtain time-dispersion diversity. However, this characterization was only applicable to signals with very high bandwidth as it assumed that the transmitted waveform was an impulse. In [2], we presented a more generalized characterization that was applicable to a larger class of signals by using the normal-mode model in [3] assuming perfect waveguide conditions (homogeneous fluid layer with a soft top and rigid seabed).

Note, however, that both of the above methods require accurate environmental information (such as bathymetry, sound speed profile, attenuation and density), which is often unavailable or inaccurate, in order to obtain the closed form expressions of the models. In this paper, we employ a blind method for separating the time-frequency (TF) components of the received signal that relate to some nonlinear functions. This technique consists of two steps: (i) the TF structures of the received signal are identified [4], and (ii) the TF components are separated using a TF based non-unitary warping technique [5]. After the separation of each component, we design a pilotaided communication scenario, and we obtain time-dispersion diversity by appropriate receiver design. Note that we conduct our investigation in the context of the Pekeris waveguide model with pressure-release surface and fluid boundaries [6], which is a simplified model for shallow water environments.

\section{Shallow Water EnVironment Modeling}

The Perkeris model treats the shallow water environment with pressure-release surface and a fluid seabed following [3], [6], [7]. This is shown in Fig. 1 using the coordinate system $(r, z)$ corresponding to range and depth, respectively. An omnidirectional point source with spectrum $X(f)$ is located in the ocean at $r=0$ and $z=z_{0}$. We consider the sound speed in the ocean as a constant $c \mathrm{~m} / \mathrm{s}$ and density $\rho \mathrm{kg} / \mathrm{m}^{3}$, and at the seabed as $c_{B} \mathrm{~m} / \mathrm{s}$ and density $\rho_{B} \mathrm{~kg} / \mathrm{m}^{3}$.

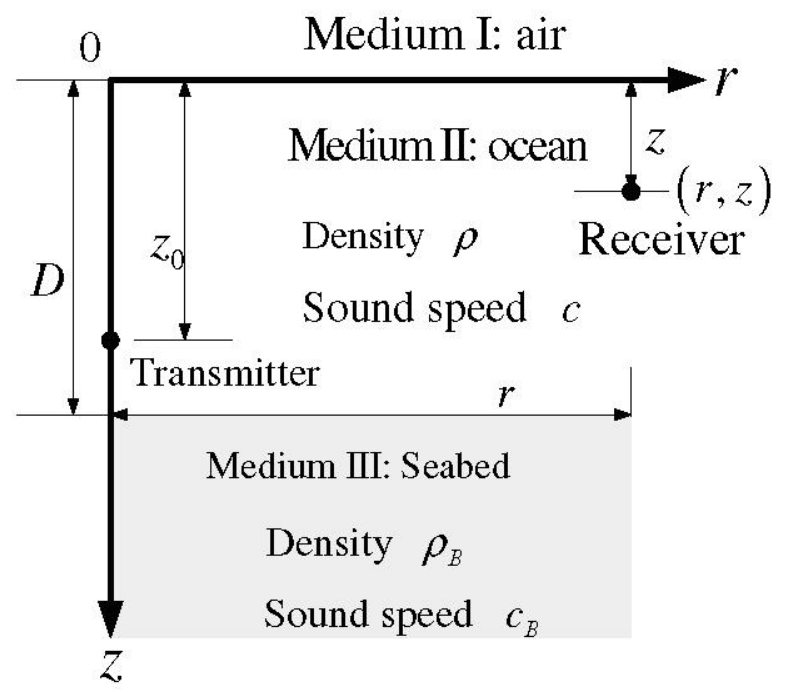

Fig. 1. Waveguide model with point source in Medium II at $r=0, z=z_{0}$, $D \mathrm{~m}$ deep for a pressure release surface with a fluid seabed [6].

The ocean surface (at $z=0$ ) is modeled realistically as an ideal pressure release boundary, and the ocean bottom (at $z=D$ ) is modeled as a boundary between two different fluid media. The normal mode model is given by the solution of this ocean waveguide problem, which is determined by the 
environment parameters and satisfies all boundary conditions, including the boundary condition at the source.

Following [6] and [7], the received signal spectrum excited by $X(f)$ at $(r, z)$ is given by the Pekeris waveguide model:

$$
Y_{\text {Pekeris }}(f)=X(f) \sum_{n=0}^{N_{m}-1} C_{n}(f) \Theta_{n}(f) .
$$

Without the assumption of ideal waveguide condition as in [2], the $n$th mode is characterized by

$$
\Theta_{n}(f)=\sqrt{\frac{1}{k_{n}(f) r}} e^{-j k_{n}(f) r},
$$

where

$$
k_{n}(f)=\frac{2 \pi}{c}\left(f^{2}-\frac{n^{2} c^{2}}{4 D^{2}}\left(1+\frac{\rho_{B} c}{\rho D} \frac{1}{2 \pi f \sqrt{1-\frac{c^{2}}{c_{B}^{2}}}}\right)^{-2}\right)^{\frac{1}{2}}
$$

is the wave number of the $n$th mode and $N_{m}$ is the largest mode number. The parameter $C_{n}(f)=$ $A_{n}^{2}(f) \sin \left(k_{z n}(f) z_{0}\right) \sin \left(k_{z n}(f) z\right)$ in (1) is a function of frequency where

$$
\begin{aligned}
A_{n}(f) & =\sqrt{2}\left[\frac{1}{\rho}\left(D-\frac{\sin \left(2 k_{z n}(f) D\right)}{2 k_{z n}(f)}\right)\right. \\
& \left.-\frac{\rho}{\rho_{B}^{2}} \frac{\tan \left(k_{z n}(f) D\right) \sin ^{2}\left(k_{z n}(f) D\right)}{k_{z n}(f)}\right]^{-\frac{1}{2}}
\end{aligned}
$$

and $k_{z n}(f) \approx \frac{n \pi}{D}\left(1+\frac{\rho_{B} c}{\rho D} \frac{1}{2 \pi f \sqrt{1-\frac{o^{2}}{o_{B}^{2}}}}\right)^{-1}$.

The TF characteristic of the acoustic signal is determined by the modal group velocity (MGV) $g_{n}(f)$, and the propagating delay of the frequency $f_{0}$ in the $n$th mode is determined by $\tau_{n}\left(f_{0}\right)=\frac{r}{g_{n}\left(f_{0}\right)}$. The MGV is shown in Fig. 2. Note that, for the waveguide model with fluid seabed, the MGV approaches $c$ when the frequency approaches infinity; it approaches $c_{B}$ when the frequency approaches the cutoff frequency of this mode.

\section{BLIND IDENTIFICATION AND SEPARATION}

In many cases, environmental parameters such as TF characteristics are not known and need to be identified. In this section, we are considering the problem of identifying the TF structures (or group velocity signatures) of the received signal after it propagates through shallow water, and then separate the TF components without knowing the environmental parameters.

\section{A. Blind Identification of Normal Modes}

Following the normal-mode model in [3], we expect the TF structures to vary dispersively with frequency according to some functions $\xi_{n}(f)=k_{n}(f) r, n=0, \cdots, N_{m}-1$ in (1). The normal-mode model treats the ocean as a waveguide with plane, parallel boundaries, representing the acoustic field in the ocean medium as a sum of normal modes; in the

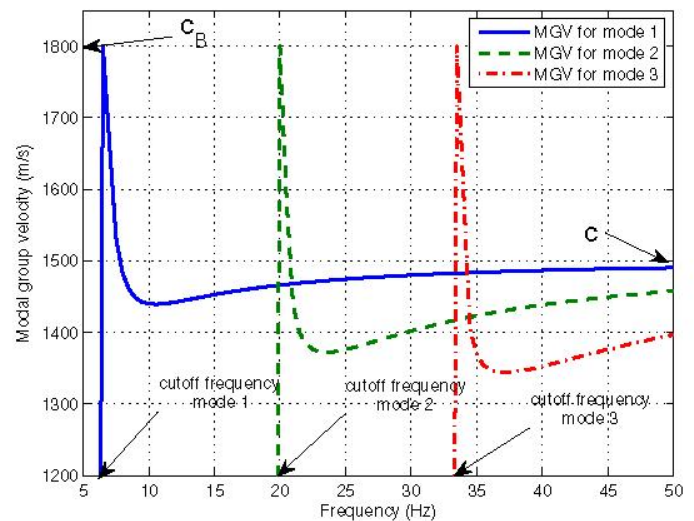

Fig. 2. Modal group velocity (MGV) function.

context of this paper, each mode can be seen as a TF signature component. For example, Fig. 3(a) represents the spectrogram of the received signal for $N_{m}=3$ modes where the ocean depth is $100 \mathrm{~m}$ and the range between transmitter and receiver is $15 \mathrm{~km}$. Each mode is shown as a dispersive component in the TF plane.

Specifically, following (1), we denote the $n$th mode as

$$
Y_{n}(f)=C_{n}(f) \Theta_{n}(f) .
$$

We want to approximate the real part of $Y_{n}(f)$ for $f \in$ $[i \Delta f,(i+1) \Delta f], i=0, \cdots, I_{n}-1$ using the linear chirps $\bar{Y}_{n, i}(f)=\cos \left(2 \pi \phi_{n, i}(f)\right)$. Here $\Delta f$ is a fixed frequency interval, $I_{n}$ is the total number of chirps used to approximate the $n$th signature, and

$$
\phi_{n, i}(f)=a_{n, i}+b_{n, i} f+c_{n, i} f^{2},
$$

where $a_{n, i}, b_{n, i}$ and $c_{n, i}$ are the chirp parameters. Since the group velocity function (GVF) in (4) is a continuous function, We expect that the chirp $\bar{Y}_{n, i}(f)$ is related to the chirp $\bar{Y}_{n, i+1}(f)$ by the following continuity constraints. Since chirp sequence has continuous instantaneous frequency and phase, then

$$
\begin{gathered}
b_{n, i+1}=b_{n, i}+2(i \Delta f)\left(c_{n, i}-c_{n, i+1}\right), \\
a_{n, i+1}=a_{n, i}+b_{n, i}(i \Delta f)+c_{n, i}(i \Delta f)^{2} .
\end{gathered}
$$

Let $\bar{Y}_{n, i}(f)$ be the chirp which best approximates the component $Y_{n}(f)$ over the frequency interval $[i \Delta f,(i+1) \Delta f)$. Then the next chirp $\bar{Y}_{n, i+1}(f)$ on the frequency interval $[(i+1) \Delta f,(i+2) \Delta f)$ is defined to be the one that best matches $Y_{n}(f)$ with the continuity constraints (5) and (6).

The problem of finding $\bar{Y}_{n, i}(f)$ can be formulated as a multi-hypothesis detection problem, which can be solved by quadrature matched filtering [8]. We denote the candidates for the chirp phase as $\phi_{n, i}^{k}(f), k=1, \cdots, K$. The best of the candidates, $\phi_{n, i}^{k_{\text {best }}}(f)$, can be found by the following 
maximization problem

$$
k_{\text {best }}=\max _{k} \frac{n_{s}^{k}\left(x_{c}^{k}\right)^{2}-2 n_{c s}^{k} x_{c}^{k} x_{s}^{k}+n_{c}\left(x_{s}^{k}\right)^{2}}{2 n_{c}^{k} n_{x}^{k}-2\left(n_{c s}^{k}\right)^{2}},
$$

subjected to (5) and (6), where

$$
\begin{gathered}
x_{c}^{k}=\int_{i \Delta f}^{(i+1) \Delta f} Y_{n}(f) \cos \left(2 \pi \phi_{n, i}^{k}(f)\right) d t, \\
x_{s}^{k}=\int_{i \Delta f}^{(i+1) \Delta f} Y_{n}(f) \sin \left(2 \pi \phi_{n, i}^{k}(f)\right) d t, \\
n_{c}^{k}=\int_{i \Delta f}^{(i+1) \Delta f} \cos ^{2}\left(2 \pi \phi_{n, i}^{k}(f)\right) d t, \\
n_{s}^{k}=\int_{i \Delta f}^{(i+1) \Delta f} \sin ^{2}\left(2 \pi \phi_{n, i}^{k}(f)\right) d t, \\
\text { and } n_{c s}^{k}=\int_{i \Delta f}^{(i+1) \Delta f} \cos \left(2 \pi \phi_{n, i}^{k}(f)\right) \sin \left(2 \pi \phi_{n, i}^{k}(f)\right) d t .
\end{gathered}
$$

Thus, the solution to the above constrained programming problem can give the optimal estimation of $Y_{n}(f)$ for $f \in$ $[i \Delta f,(i+1) \Delta f)$ in additive white Gaussian noise. Using this approach, the dispersive curves in Fig. 3(a) are estimated in Fig. 3(b).

\section{B. Time-Frequency Component Separation}

Fig. 3(a) illustrates that each mode of the received signal appears as a dispersive curve in the TF plane, which makes the separation of the modes possible. In this section, we use a TF mode separation technique based on a warping technique.

In Section III-A, we approximated $N_{m}$ normal modes. We can then design $N_{m}-1$ GVF curve separators in the TF plane, represented as $e_{n}(f), n=0, \cdots, N_{m}-2$. A GVF curve separator is a curve situated between two successive modes in the TF plane as shown in Fig. 3(c). Knowing the TF signatures, we appropriately set $M$ TF points $\left(t_{l}, f_{l}\right), l=1,2, \ldots, M$ in the middle of the space between two successive modes. The $M$ points between the $n$th mode and the $(n+1)$ th mode will constitute the GVF curve separator $e_{n}(f)$ that will be used for separating the $N_{m}$ TF signatures $Y_{n}(f), n=0, \cdots, N_{m}-1$.

To separate the TF components according to the obtained GVF curve separators, we employ a non-unitary warping operation defined as

$$
\left(\check{\mathcal{W}}_{\zeta} Y\right)(f)=\int_{\mathbb{R}} Y(v) \delta\left(v-\zeta^{-1}(f)\right) d v=Y\left(\zeta^{-1}(f)\right) .
$$

The non-unitary operator overcomes the spreading effect of unitary warping operators [5], and it can be verified that

$$
\left(\mathcal{F}^{-1} \mathscr{\mathcal { W }} e^{-j 2 \pi t_{0} \zeta(f)}\right)(t)=\delta\left(t-t_{0}\right)
$$

as $\left(\breve{\mathcal{W}}_{\zeta} e^{-j 2 \pi t_{0} \zeta(f)}\right)(f)=e^{j 2 \pi f t_{0}}$, where the inverse Fourier transform $(\mathrm{FT})$ is given by $\left(\mathcal{F}^{-1} X(f)\right)=x(t)$.

We process the different modes in the TF plane using this non-unitary warping [4], [5] and the local harmonic convolution operator [5]. Assume that the received noisy signal in (1) is $r(t)$ with FT $R(f)=Y_{\text {Pekeris }}(f)+W(f)$, where
$W(f)$ is additive white Gaussian noise. We then obtain the warping functions from the GVF curve separators as $\zeta_{n}(f)=$ $\frac{1}{\lambda_{r}} \int_{-\infty}^{f} e_{n}(v) d v$, where $\lambda_{T}>0$ is a normalization constant, and compute the corresponding generalized FT of $R(f)$ as [9]

$$
M_{R}(\lambda)=\left(\mathcal{M}_{\zeta_{n}} R\right)(\lambda)=\int_{\wp} R(f) \frac{d \zeta_{n}(f)}{d f} e^{j 2 \pi \lambda \zeta_{n}(f)} d f
$$

Here, $\wp$ contains the values of $f$ in the domain of the warping function $\zeta_{n}(f)$, and $\lambda$ is a real and unitless parameter. To obtain the first mode $n=0$, we compute the inverse generalized FT over the range $\left(-\infty, \lambda_{T}\right]$ as follows:

$$
R_{0}(f)=\int_{-\infty}^{\lambda_{r}} M_{R_{0}}(\lambda) e^{-j 2 \pi \lambda \zeta_{0}(f)} d \lambda
$$

Further, we can rewrite (11) as

$$
R_{0}(f)=\int_{-\infty}^{\infty} h_{L}(\lambda) \mathcal{M}_{R_{0}}(\lambda) e^{-j 2 \pi \lambda \zeta_{0}(f)} d \lambda
$$

where $h_{L}(\lambda)=1$ if $\lambda \in\left(-\infty, \lambda_{r}\right]$ and $h_{L}(\lambda)=0$ if $\lambda \epsilon$ $\left(\lambda_{r}, \infty\right]$. One can show that (12) simplifies to

$$
\begin{aligned}
R_{0}(f) & =\int_{-\infty}^{\infty} \int_{\wp} R(v) h_{L}(\lambda) \frac{d \zeta_{0}(v)}{d v} e^{j 2 \pi \lambda\left(\zeta_{0}(v)-\zeta_{0}(f)\right)} d v d \lambda \\
& =\int_{\wp} R(v) \frac{d \zeta_{0}(v)}{d v} H_{L}\left(\zeta_{0}(f)-\zeta_{0}(v)\right) d v
\end{aligned}
$$

where $H_{L}(f)=\int_{-\infty}^{\infty} h_{L}(\lambda) e^{-j 2 \pi \lambda f} d \lambda$ is the FT of the lowpass filter $h_{L}(\lambda)$.

We subtract the first mode from the received signal, i.e, $\hat{R}(f)=R(f)-R_{0}(f)$, and the remaining modes are contained in $\hat{R}(f)$. To obtain the seconde mode, we apply the above procedure to $\hat{R}(f)$ using the GVF curve separator $e_{1}(f)$. Repeating the above procedure for each GVF curve separator, we can then separate each mode from the received signal.

For the example in Fig. 3, we use the mode separation technique on the received signal excited by the waveform $X(f)=\sqrt{f}, f>0$ for $N_{m}=3$ modes. The GVF curve separators are shown in Fig. 3(c) and the separated components are shown in Fig. 3(d).

\section{TIME-DISPERSION DIVERSITY RECEIVER DESIGN}

Although the shallow water environment model provides an inherent frequency domain transfer function, in realistic shallow water environments, many factors can cause distortion in the signal propagation. For example, the ocean surface fluctuates with the waves, and the roughness of the ocean bottom affects the signal reflections. Hence, it is reasonable to introduce randomness into the channel model. We model this distortion following the data generated by the normal-mode modeling software KRAKEN [10]. Specifically, we model the randomness by $D_{n}$ which can be modeled as

$$
D_{n}=\alpha_{n}+\Delta \varrho_{n} \text {. }
$$

This is a random variable with mean $\alpha_{n}$ and variance $\sigma_{D_{n}}^{2}$. In practice, $D_{n}$ needs to be measured by conducting a system identification. Additive noise is also introduced in the model 


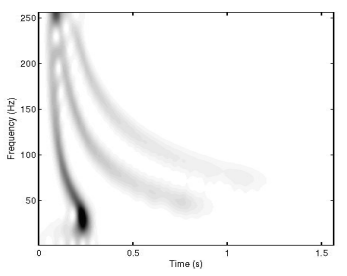

(a)

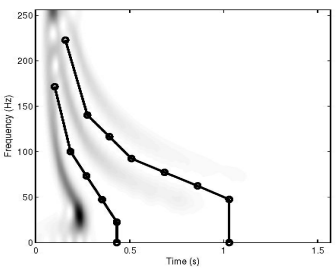

(c)

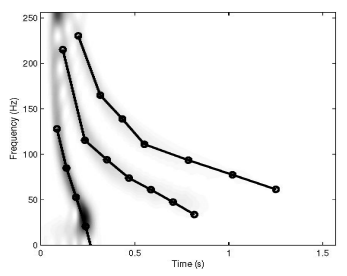

(b)

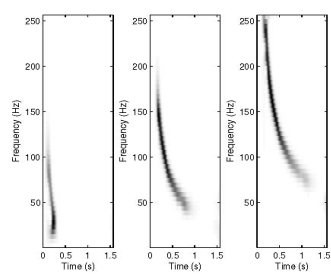

(d)
Fig. 3. (a) Spectrogram of received signal. (b) Approximated TF signatures (GVFs). (c) GVF curve separators. (d) Separated TF components.

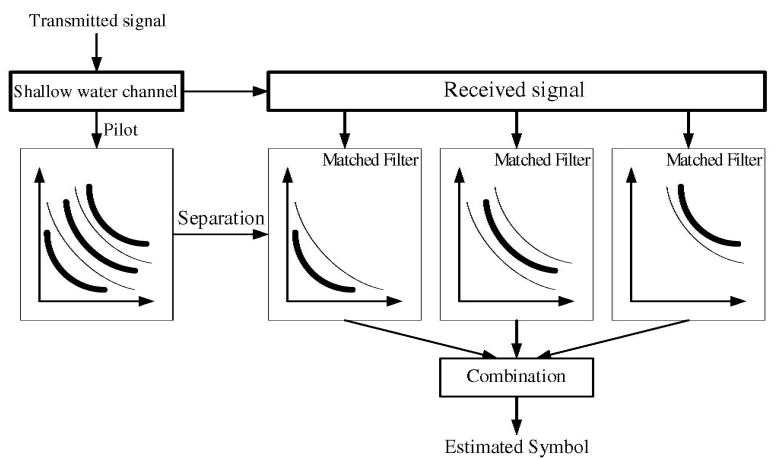

Fig. 4. A receiver design for time-dispersion diversity using TF mode separation.

due to the random disturbance in the ocean environment and receiver. Hereby, the received signal spectrum is expressed as

$$
R(f)=Y(f)+W(f)=X(f) \sum_{n=0}^{N_{m} 1} D_{n} C_{n}(f) \Theta_{n}(f)+W(f),
$$

$W(f)$ is additive white Gaussian noise with variance $\sigma_{W}^{2}$.

\section{A. Receiver Design with TF Component Separation}

After the TF mode-components are separated, each component can be treated as a subchannel of the shallow water communication channel; hence diversity can be obtained if the receiver is properly designed. Our proposed receiver design for diversity is shown in Fig. 4. To exploit the potential diversity, we first transmit a pilot symbol $b$ to separate the modes. Then, the received signal is analyzed, and each component is separated and used jointly with the channel coefficients as the matched filter for the received signal of the next transmitted symbols. The outputs of the matched filters are combined, and the decisions for the estimated symbols are made using a minimum error probability detector.
This receiver processing can be described as follows. Concatenating the signals $U_{n}(f)=C_{n}(f) \Theta_{n}(f)$, we can express the resulting separation results using vector $\mathbf{U}(f)=$ $\operatorname{diag}\left\{U_{0}(f), \cdots, U_{N_{m}-1}(f)\right\}$. Similarly, we rewrite the transmitted symbol $b$ as an $N_{m} \times 1$ vector $\mathbf{b}=[b, b, \cdots b]^{\mathbb{T}}$. Let $\mathbf{D}=\operatorname{diag}\left(D_{0}, D_{1}, \cdots, D_{N_{m}-1}\right)$ be the $N_{m} \times N_{m}$ matrix whose diagonal elements are the random channel coefficients in (14), and $\mathbf{W}(f)=\left[W_{0}(f), \cdots, W_{N_{m}-1}(f)\right]^{\mathbb{T}}$, where we assume a different noise covariance for each TF component after separation. Using the vector notation, the received spectrum after separation can also be written as

$$
\mathbf{R}(f)=\mathbf{U}(f) \mathbf{D b}+\mathbf{W}(f) .
$$

The output of this filter bank is given by $\mathbf{Z}=\mathbf{P D b}+\mathbf{W}$, where $\mathbf{P}=\int \mathbf{U}^{*}(f) \mathbf{U}(f) d f$ is the matrix of correlations between different modes, and $\mathbf{W}=\int \mathbf{U}^{*}(f) \mathbf{W}(f)$ df is the noise at the output of the matched filters. It can be shown that the expected value $\mathrm{E}\left[W_{m}\left(f_{1}\right) W_{n}\left(f_{2}\right)\right]=0, \forall f_{1}, f_{2}$, for $m \neq n$. As a result, $\mathbf{C}_{c o v}=\mathrm{E}\left[\mathbf{W} \mathbf{W}^{\dagger}\right]=\operatorname{diag}\left\{\sigma_{0}^{2}, \cdots, \sigma_{N_{m}-1}^{2}\right\}$ is a diagonal matrix, where $\sigma_{n}^{2}$ is the noise covariance in the $n$th subchannel.

If binary antipodal symbols are transmitted, i.e., $b=+1$ or -1 , and we denote $\mathbf{d}=\mathbf{D b}$ using the above notation, then the communication problem stated above is converted to a classic, detection problem with hypothesis

$$
\begin{aligned}
& \mathcal{H}_{0}: \mathbf{Z}=-\mathbf{P d}+\mathbf{W} \\
& \mathcal{H}_{1}: \mathbf{Z}=\mathbf{P d}+\mathbf{W} .
\end{aligned}
$$

We notice that $\mathbf{P}$ is a diagonal matrix whose elements correspond to the powers of each mode $q_{n}, n=0, \ldots, N_{m}-1$. To compute $q_{n}$, we can transmit two pilot symbols, and after separation, we can obtain $D_{n}^{(1)} U_{n}^{(1)}(f)+W_{n}^{(1)}(f)$ and $D_{n}^{(2)} U_{n}^{(2)}(f)+W_{n}^{(2)}(f)$, respectively, for two transmissions. We already assumed $D_{n}^{(1)}$ and $D_{n}^{(2)}$ are known. Thus, $q_{n} \approx$ $\int\left(D_{n}^{(1)} U_{n}^{(1)}(f)+W_{n}^{(1)}(f)\right)\left(D_{n}^{(2)} U_{n}^{(2)}(f)+W_{0}^{(2)}(f)\right)^{*} d f$, because $W_{0}^{(1)}(f)$ and $W_{0}^{(2)}(f)$ are zero mean and uncorrelated. We also know that all the elements are greater or equal to zero. Hereby we assume $q_{0} \geq q_{1} \geq \cdots q_{N_{m}-2} \geq q_{N_{m}-1} \geq 0$. Then according to the Neyman-Pearson theorem, the detector decides $\mathcal{H}_{1}$ if

$$
\mathrm{L}(\mathbf{Z})=\frac{\prod_{n=0}^{N_{m}-1} e^{-\frac{\left|Z_{n}-q_{n} d_{n}\right|^{2}}{2 \sigma_{n}^{2} q_{n}}}}{\prod_{n=0}^{N_{m}-1} e^{-\frac{\left|Z_{n}+q_{n} d_{n}\right|^{2}}{2 \sigma_{n}^{2} q_{n}}}}>\gamma,
$$

where $\mathrm{L}(\mathbf{Z})$ is the likelihood function for this detection problem, $Z_{n}$ is the $n$th element of vector $\mathbf{Z}$, and $d_{n}$ is the $n$th element of vector $\mathbf{d}$. The threshold $\gamma$ is computed using the Bayesian approach to minimize the probability of error in the received symbols. If the probabilities of transmitting +1 and -1 are equal, we can choose $\gamma=1$ to obtain the minimum bit error rate (BER). After simplification of (19), the detector decides $\mathcal{H}_{1}$ if

$$
\sum_{n=0}^{N_{m}-1} \Re\left\{\frac{Z_{n} d_{n}^{*}}{\sigma_{n}^{2}}>0\right\}
$$


where $\Re\{\cdot\}$ is the operator that takes the real part.

\section{B. Performance Analysis}

In this section, the BER and diversity performances of the proposed receiver design are investigated. From (16), we know that the $N_{m}$ correlated received signals can be transformed into $N_{m}$ independent received signals. Without loss of generality, we assume that $b=1$ is transmitted. Then, the $N_{m}$ independent signals can be expressed as $Z_{n}=$ $q_{n} d_{n}+W_{n}, \quad n=0,1, \cdots N_{m}-1$, where $Z_{n}$ is the $n$th element of $\mathbf{Z}$, and $W_{n}$ is the $n$th element of noise $\mathbf{W}$. According to the minimum error probability detector rule in (20), we have

$$
Z_{n} d_{n}^{*}=q_{n} d_{n} d_{n}^{*}+W_{n} d^{*}, \quad n=0,1, \cdots N_{m}-1,
$$

The SNR of the $n$th received signal in (21), denoted as $\eta_{n}$, can be expressed as

$$
\eta_{n}=\frac{q_{n}^{2}\left|d_{n}\right|^{4}}{\mathrm{E}\left[d_{n} W_{n}^{*} W_{n} d_{n}^{*}\right]}=\frac{q_{n}^{2}\left|d_{n}\right|^{4}}{q_{n} \sigma_{n}^{2}\left|d_{n}\right|^{2}}=\frac{q_{n}\left|d_{n}\right|^{2}}{\sigma_{n}^{2}} .
$$

From (14), we know that the $n$th component of $\mathbf{d}$ can be expressed as $D_{n}=\alpha_{n}+\Delta \varrho_{n}$. If we reasonably assume that $\Delta \varrho_{n}$ is independent for each mode, the covariance matrix of d can be given by

$$
\begin{aligned}
\mathbf{C}_{D D} & =\mathrm{E}\left[(\mathbf{d}-\mathrm{E}[\mathbf{d}])(\mathbf{d}-\mathrm{E}[\mathbf{d}])^{\dagger}\right] \\
& =\operatorname{diag}\left\{\sigma_{D_{0}}^{2}, \sigma_{D_{1}}^{2}, \cdots, \sigma_{D_{N_{m}-1}}^{2}\right\} .
\end{aligned}
$$

If we define $\boldsymbol{\Sigma}=\mathbf{C}_{c o v}^{-\frac{1}{2}} \mathbf{C}_{D D} \mathbf{C}_{c o v}^{-\frac{1}{2}}$, then from (22) and (23), we know that $\boldsymbol{\Sigma}$ is full rank. Using the minimum error probability detector rule in (20), the average BER is [11]:

$$
P_{b}=\frac{1}{\pi} \int_{0}^{\frac{\pi}{2}}\left[\operatorname{det}\left(\frac{\boldsymbol{\Sigma}}{\sin ^{2} \theta}+\mathbf{I}\right)\right]^{-1} e^{-\mathbf{m}^{\dagger}\left(\boldsymbol{\Sigma}+\sin ^{2} \theta \mathbf{I}\right)^{-1} \mathbf{m}} d \theta,
$$

where $\mathbf{m}=\mathbf{C}_{c o v}^{-\frac{1}{2}} \mathrm{E}[\mathbf{d}]$. The potential diversity order is given by $N_{m}$.

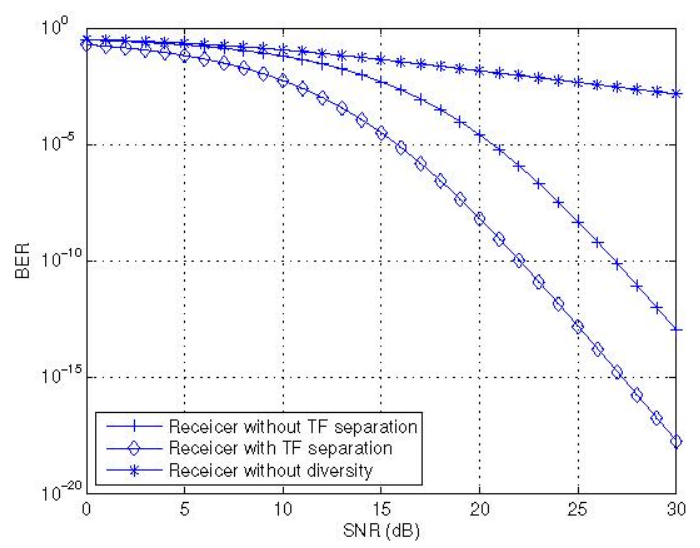

Fig. 5. BER and diversity performance in different transmission bands.

The BER numerical results for varying SNR are shown in Fig. 5. Here we used the environment parameters $D=100$ $\mathrm{m}, c=1500 \mathrm{~m} / \mathrm{s}$ and $r=15 \mathrm{~km}$. The numerical results show the BER and diversity performances of three different types of receivers: receiver with TF component separation which is designed in this paper, receiver without TF component separation which is designed in [2], and receiver without diversity which is a conventional matched filter. As we can see, the BER performance of the receiver with the separated TF components outperforms the other two. This is because this receiver avoids interference between normal modes. Furthermore, the separation performs the TF denoising of the received signal, which further improved the SNR.

\section{CONCLUSION}

We investigated the frequency domain characterization of shallow water environments and analyzed its dispersive characteristics based on the Pekeris waveguide model. Following this model, we developed a new method, based on a warping technique, for the blind separation of the TF modecomponents, adaptively determining the group velocity curves and filtering the TF components of the received signal. As an application example, we developed the corresponding waveform and receiver to exploit the diversity existing in the system characterization. Numerical results demonstrated that the diversity and BER performances were improved by the aforementioned receiver design scheme.

\section{ACKNOWLEDGMENT}

This work was supported by the NSF CAREER Award CCR-0134002 and the Department of Defense MURI Grant No. AFOSR FA9550-05-1-0443.

\section{REFERENCES}

[1] Y. Jiang and A. Papandreou-Suppappola, "Discrete time-frequency characterizations of dispersive linear time-varying systems," IEEE Trans. Signal Processing, vol. 55, pp. 2066-2076, May 2007.

[2] J. Zhang and A. Papandreou-Suppappola, "Time-frequency based waveform and receiver design for shallow water communications," in Proc. IEEE Int. Conf. on Acoust., Speech, Signal Processing, vol. 3, April 2007, pp. 1149-1152.

[3] L. J. Ziomek, Fundamentals of Acoustic Field Theory and Space-Time Signal Processing. CRC Press, 1999.

[4] A. Jarrot, C. Ioana, C. Gervaise, and A. Quinquis, "A time-frequency characterization framework for signals issued from underwater dispersive environments," in Proc. IEEE Int. Conf. on Acoust., Speech, Signal Processing, vol. 3, April 2007, pp. 1145-1148.

[5] A. Jarrot, C. Ioana, and A. Quinquis, "A class of linear time-varying filters based on non-unitary time-warping operators. Part I: principle and examples," submitted to IEEE Trans. Signal Processing, 2006.

[6] G. V. Frisk, Ocean and Seabed Acoustics. Prentice-Hall, 1994.

[7] G. B. Deane, "Internal friction and boundary conditions in lossy fluid seabeds," The Joumal of the Acoustical Society of America, vol. 101, no. 1, pp. 233-240, January 1997.

[8] É. Chassande-Mottin and A. Pai, "Best chirplet chain: Near-optimal detection of gravitational wave chirps," Physical Review, vol. 73, no. 042003 , pp. 1-25, Feb. 2006.

[9] A. Papandreou-Suppappola, "Time-varying processing: Tutorial on principles and practice," in Applications in Time-Frequency Signal Processing (A. Papandreou-Suppappola, ed.). Florida: CRC Press, 2002.

[10] A. L. Maggi and A. J. Duncan, "Underwater acoustic propagation modelling software," http://www.cmst.curtin.edu.au/products/actoolbox.

[11] V. Veeravalli, "On performance analysis for signaling on correlated fading channels," IEEE Trans. Commun., vol. 49, no. 11, pp. 1879 1883, Nov. 2001. 